\title{
On an asymptotic formula of Srinivasa Ramanujan
}

\author{
by
}

K. Ramachandra (Bangalore) and A. Sankaranarayanan (Mumbai)

To Professor R. Balasubramanian on his fiftieth birthday

1. Introduction. In [16], Ramanujan records (without proof) many curious asymptotic formulae. One of them is

$$
\begin{aligned}
d^{2}(1)+d^{2}(2)+\ldots+d^{2}(n)= & A n(\log n)^{3}+B n(\log n)^{2}+C n \log n \\
& +D n+O\left(n^{3 / 5+\varepsilon}\right) .
\end{aligned}
$$

Also he records (without proof) the result that on the assumption of the Riemann hypothesis, the error term in (1.1) can be improved to $O\left(n^{1 / 2+\varepsilon}\right)$. In view of a method due to H. L. Montgomery and R. C. Vaughan (see [9]), it is very likely that the error term is $O\left(n^{1 / 2}\right)$. We propose this as a conjecture (see also [15], [17]). Unconditionally, the error term related to $d^{2}(j)$ is known to be $O\left(n^{1 / 2+\varepsilon}\right)$ for any positive constant $\varepsilon$ (see for example the equation (14.30) of [6] and also [5]). Professor A. Schinzel has already considered some of the problems of Ramanujan (see [19]), namely for the arithmetic function $r^{2}(n)$, and he has proved that the corresponding error term is $\Omega\left(n^{3 / 8}\right)$ and also the corresponding error term is $O\left(n^{1 / 2}(\log n)^{8 / 3}(\log \log n)^{1 / 3}\right)$ due to an unpublished work of W. G. Nowak (see also [8] and [18]). Let

$$
E(x)=\sum_{n \leq x} d^{2}(n)-x P_{3}(\log x)
$$

where $P_{3}(y)$ is a polynomial in $y$ of degree 3 . From a general theorem of M. Kühleitner and W. G. Nowak (see for example (5.4) of [8]), it follows that

$$
E(x)=\Omega\left(x^{3 / 8}\right) .
$$

From Vinogradov's estimate (for $T / 2 \leq t \leq T$ )

2000 Mathematics Subject Classification: Primary 11M; Secondary 11M06, 11N05.

Key words and phrases: error term, Perron's formula, Riemann zeta-function, fourth power mean, Vinogradov's estimate. 


$$
\frac{1}{\zeta(1+i t)} \ll(\log T)^{2 / 3}(\log \log T)^{1 / 3},
$$

it is not very difficult to prove

TheOREM A. We have

$$
E(x)=O\left(x^{1 / 2}(\log x)^{17 / 3}(\log \log x)^{1 / 3}\right) .
$$

REMARK. We note here that an analogue of Theorem A for the "sums of two squares" function $r(n)$ was dealt with by M. Kühleitner (see [7]). We also refer to the related papers [2], [3], [12] and [20].

On the assumption of the quasi-Riemann hypothesis (namely $\zeta(s) \neq 0$ for $\sigma>\alpha$ with $1 / 2<\alpha<1$ ), following the proof of Theorems 14.6 and 14.8 of [21], we obtain the inequality

$$
\frac{1}{|\zeta(1+2 i t)|} \ll_{\alpha} \log \log t
$$

Hence one gets have

COROLlary. On the assumption of the quasi-Riemann hypothesis, we

$$
E(x)=O\left(x^{1 / 2}(\log x)^{5}(\log \log x)\right) .
$$

The main goal of this paper is to prove

MAIN TheOREM. Unconditionally, we have

$$
E(x)=O\left(x^{1 / 2}(\log x)^{5}(\log \log x)\right) .
$$

REMARK. It is not difficult to prove an ineffective result like

$$
E(x)=\Omega_{ \pm}\left(x^{1 / 4}\right) .
$$

The ineffective version is due to E. Landau (see [4]). The general method of proving results like the one above (actually in an effective way) is due to R. Balasubramanian and K. Ramachandra (see [1]).

2. Notation and preliminaries. $C$ and $A$ (with or without subscripts) denote effective positive constants unless specified otherwise; $\varepsilon$ will always denote a sufficiently small positive constant; $T \geq T_{0}$ (a sufficiently large positive constant). We write $f(x) \ll g(x)$ to mean $|f(x)|<C_{1} g(x)$ (sometimes we denote this by the $O$ notation also). Let $s=\sigma+i t, s_{0}=1 / 2+i t$ and $w=u+i v$. The notation $[x]$ denotes the integral part of $x$ whereas $[a, b]$ denotes the interval $a \leq c \leq b$. The implied constants are all effective.

\section{Some lemmas}

Lemma 3.1 (Refined version of Perron's formula). Let $\left\{\lambda_{n}\right\}$ be a sequence of real numbers with $0<\lambda_{1}<\ldots<\lambda_{n} \rightarrow \infty$ and $\left\{a_{n}\right\}$ be any se- 
quence of complex numbers such that $f(s)=\sum_{n=1}^{\infty} a_{n} \lambda_{n}^{-s}$ (with $s=\sigma+i t$ ) is absolutely convergent in $\sigma>1$. Then for $x>0, C>1$, we have uniformly (in all the parameters) the equality

$$
\begin{aligned}
\frac{1}{T} \int_{0}^{T} \ldots \frac{1}{T} \int_{0}^{T}\left(\frac{1}{2 \pi i} \int_{C-i T-i \phi}^{C+i T+i \phi} f(s) \frac{x^{s}}{s} d s\right) d \tau_{1} \ldots d \tau_{k} \\
\quad=\sum_{\lambda_{n} \leq x} a_{n}+\frac{\theta}{\pi} \sum_{n=1}^{\infty}\left|a_{n}\right|\left(\frac{x}{\lambda_{n}}\right)^{C} \min (\pi+2+C / T, M)
\end{aligned}
$$

where

$$
M=\min _{0 \leq m \in \mathbb{Z}^{+} \leq k}\left(\frac{2^{m+1}-1}{m+1}\right)\left|T \log \left(\frac{x}{\lambda_{n}}\right)\right|^{-m-1},
$$

$\theta$ is a complex number with $|\theta| \leq 1$ (moreover $\theta$ is real if $a_{n}$ are all real) and $T>0, \tau_{1}, \ldots, \tau_{k}$ are real variables with $0 \leq \tau_{j} \leq T(j=1, \ldots, k)$, $\phi=\tau_{1}+\ldots+\tau_{k}$ (we define an empty sum as zero).

Proof. See Corollary 2 of [14].

Lemma 3.2. Let $T / 2<t_{1}<\ldots<t_{R} \leq T$ be well spaced points satisfying $\left|t_{j+1}-t_{j}\right| \geq 1$ (for $\left.j=1, \ldots, R-1\right)$, and suppose that for every small positive constant $\varepsilon$, the points $t_{j}$ satisfy the inequality

$$
\left|\log \zeta\left(1+i t_{j}\right)\right| \gg \log \log \log T-10 \log \varepsilon .
$$

Then

$$
R \ll T^{2 \varepsilon} .
$$

Remark. This is Theorem 1 of [13]. For the sake of completeness, we present here a simple proof of Lemma 3.2.

Proof of Lemma 3.2. First of all we note that from the density estimates, we have (see [6])

$$
N(\sigma, T, 2 T) \ll T^{\frac{12}{5}(1-\sigma)}(\log T)^{100} .
$$

Let $\delta$ be a small positive constant, say $0<\delta<1 / 100$. Suppose that the number $N(1-\delta, T, 2 T)$ of zeros of $\zeta(s)$ in $\{\sigma \geq 1-\delta, T \leq t \leq 2 T\}$ is $<T^{\eta}$ where $\eta>0$ is a small positive constant (may depend on $\delta$ ). Let $\varrho=\beta+i \gamma$ be any of these zeros. With each such zero, we associate the rectangle

$$
\left\{\sigma \geq 1-\delta, t \in\left(\gamma-(\log T)^{100}, \gamma+(\log T)^{100}\right)\right\}
$$

Let $s$ be any point in the complement in $\{\sigma \geq 1-\delta, T \leq t \leq 2 T\}$ of the union of all these rectangles. (Note that we have excluded a total of $t$-height $\leq 2(\log T)^{100} T^{\eta}$ in $\{\sigma \geq 1-\delta, T \leq t \leq 2 T\}$.) From the density estimate above, we observe that the region $\left\{\sigma \geq 1-\delta, s \pm(\log T)^{100}\right\}$ is zero-free of $\zeta(s)$. Now, we can talk of $\log \zeta(s)$ in this region. If necessary, we can exclude further $\frac{1}{2}(\log T)^{100}$ on either side of this region. The total 
$t$-length thus excluded is $\leq 10(\log T)^{100} T^{\eta}$. Now, in the resulting region, we can not only talk of $\log \zeta(s)$ but even apply the Borel-Carathéodory theorem in $\sigma \geq 1-\delta / 2$ (with centres on the line $\sigma=2$ ). Therefore, in $\left\{\sigma \geq 1-\delta / 4, s \pm \frac{1}{2}(\log T)^{100}\right\}$, we have $\log \zeta(s)=O(\log T)$. Now, for $\sigma \geq 1-\delta / 8, T \leq t \leq 2 T$, we have (with $w=u+i v$ and fixing $X=(\log T)^{8 / \delta}$ )

$$
\begin{aligned}
\frac{1}{2 \pi i} \int_{\Re w=\delta / 4,|v| \leq(\log T)^{3}} \log \zeta(s & +w) \Gamma(w) X^{w} d w \\
& =\sum_{p} \frac{e^{-p / X}}{p^{s}}+O\left(\log \left(\frac{8}{\delta}\right) e^{-C(\log T)^{3}}\right) .
\end{aligned}
$$

Now, we move the line of integration in the remaining integral above to $\sigma+u=1-\delta / 4$, that is to $u=-\delta / 8$. The pole at $w=0$ of $\Gamma(w)$ gives the residue $\log \zeta(s)$. Note that our $X=(\log T)^{8 / \delta}$. The horizontal portions contribute an error which is $\ll(\log T) X^{\delta / 4} e^{-(\log T)^{3}} \ll 1$ because of the presence of the $\Gamma(w)$ in the integrand, whereas the vertical line integral on $u=-\delta / 8$ contributes an error which is $\ll(\log T) X^{-\delta / 8} \ll 1$ with our choice of $X$. Note that

$$
\sum_{p} \frac{e^{-p / X}}{p^{s}}=\sum_{p \leq X^{2}} \frac{1}{p}+O(1)=\log \log X^{2}+O(1) .
$$

Therefore we obtain

$$
\log \zeta(s)=\log \log X^{2}+O(1)+O\left(\log (8 / \delta) e^{-C(\log T)^{3}}\right)
$$

and this implies that

$$
\pm \log |\zeta(s)| \leq \log \log X^{2}+O(1)+O\left(\log (8 / \delta) e^{-C(\log T)^{3}}\right) .
$$

So, if we exclude $t$-intervals of total width $\leq T^{1000 \delta}$ on the line $\sigma=1$, for the rest, we have (for $\sigma \geq 1$ )

$$
|\zeta(\sigma+i t)|^{ \pm 1} \ll \log \log T
$$

Since $\eta$ and $\delta$ are arbitrary, this proves the lemma.

Lemma 3.3. We have (with $\left.s_{0}=1 / 2+i t\right)$

$$
x^{1 / 2} \int_{|t| \leq T}\left|\frac{\zeta^{4}\left(s_{0}\right)}{\zeta\left(2 s_{0}\right)}\right|\left|\frac{d t}{s_{0}}\right| \ll x^{1 / 2}(\log T)^{5}(\log \log T) .
$$

Proof. Let

$$
\left|\frac{1}{\zeta\left(1+2 i t_{j}\right)}\right|=\max _{j<t \leq j+1}\left|\frac{1}{\zeta(1+2 i t)}\right| .
$$

Also we have (for $T / 2 \leq t \leq T$ )

$$
\zeta(1 / 2+i t) \ll T^{1 / 6}(\log T)
$$


and

$$
\frac{1}{\zeta(1+2 i t)} \ll \log T \text {. }
$$

It is well known that (for example see [6] or [21]) for $\sigma \geq 1 / 2$,

$$
\int_{T / 2}^{T}\left|\zeta^{4}(\sigma+i t)\right| d t \ll T(\log T)^{4} .
$$

We divide the interval $[[T / 2]+1,[T]]$ into abutting small intervals of width 1 . Below, $\sum^{*}$ denotes sums over odd integers, and $\sum^{* *}$ denotes sums over even integers in the given interval.

We call a unit interval $[j, j+1] \subset[[T / 2]+1,[T]]$ a bad unit interval if

$$
\left|\log \zeta\left(1+i t_{j}\right)\right| \gg \log \log \log T-10 \log \varepsilon \text {. }
$$

From Lemma 3.2, we observe that the number of bad unit intervals in $[[T / 2]+1,[T]]$ is at most $T^{2 \varepsilon}$. For the remaining good unit intervals in $[[T / 2]+1,[T]]$, we can use the bound

$$
\frac{1}{\zeta\left(1+i t_{j}\right)} \ll \log \log T .
$$

Therefore, we obtain

$$
\begin{aligned}
& x^{1 / 2} \int_{|t| \leq T}\left|\frac{\zeta^{4}\left(s_{0}\right)}{\zeta\left(2 s_{0}\right)}\right|\left|\frac{d t}{s_{0}}\right| \\
& \ll x^{1 / 2}+x^{1 / 2} \frac{\log T}{T}\left(\int_{T / 2}^{T}\left|\frac{\zeta^{4}\left(s_{0}\right)}{\zeta\left(2 s_{0}\right)}\right| d t\right) \\
& \ll x^{1 / 2}+x^{1 / 2} \frac{\log T}{T}\left\{\sum_{j=[T / 2]+1}^{[T]-1} \int_{j}^{j+1}\left|\frac{\zeta^{4}\left(s_{0}\right)}{\zeta\left(2 s_{0}\right)}\right| d t+T^{2 / 3}(\log T)^{10}\right\} \\
& \ll x^{1 / 2}+x^{1 / 2} \frac{\log T}{T} \\
& \times\left(\left\{\sum_{j=[T / 2]+1}^{[T]-1}+\sum_{j=[T / 2]+1}^{[T]-1}\right\}_{j}^{j+1}\left|\frac{\zeta^{4}\left(s_{0}\right)}{\zeta\left(2 s_{0}\right)}\right| d t+T^{2 / 3}(\log T)^{10}\right) \\
& \ll x^{1 / 2}+x^{1 / 2} \frac{\log T}{T} \\
& \times\left(\left\{\sum_{j=[T / 2]+1}^{[T]-1}+\sum_{j=[T / 2]+1}^{* T]-1}\right\}\left|\frac{1}{\zeta\left(1+2 i t_{j}\right)}\right| \int_{j}^{j+1}\left|\zeta^{4}\left(s_{0}\right)\right| d t+T^{2 / 3}(\log T)^{10}\right)
\end{aligned}
$$


$\ll x^{1 / 2}+x^{1 / 2} \frac{\log T}{T}\left(T^{2 / 3+10 \varepsilon}(\log T)^{20}+(\log \log T)\left(\int_{[T / 2]+1}^{[T]}\left|\zeta^{4}\left(s_{0}\right)\right| d t\right)\right)$ $\ll x^{1 / 2}+x^{1 / 2}(\log T)^{5}(\log \log T)$.

This proves the lemma.

LEMMA 3.4. For $\sigma \geq 1 / 2$, we have

$\int_{1 / 2}^{1} \int_{T / 2}^{T}\left|\frac{\zeta^{4}(\sigma+i t)}{\zeta(2 \sigma+2 i t)}\right|\left|\frac{x^{s}}{s}\right| d \sigma d t \ll(\log T)^{4}(\log \log T)\left(x-x^{1 / 2}\right)(\log x)^{-1}$.

Proof. First of all we notice that by following the argument for Lemma 3.3, we obtain, for $\sigma \geq 1 / 2$,

$$
\begin{aligned}
\int_{T / 2}^{T}\left|\frac{\zeta^{4}(\sigma+i t)}{\zeta(2 \sigma+2 i t)}\right| d t & \ll \int_{T / 2}^{T}\left|\frac{\zeta^{4}(\sigma+i t)}{\zeta(1+2 i t)}\right| d t \\
& \ll(\log \log T) \int_{T / 2}^{T}\left|\zeta^{4}(1 / 2+i t)\right| d t \\
& \ll T(\log T)^{4}(\log \log T) .
\end{aligned}
$$

Therefore, from (3.4.1), we obtain

$$
\begin{aligned}
\int_{T / 2}^{T}\left|\frac{\zeta^{4}(\sigma+i t)}{\zeta(2 \sigma+2 i t)}\right|\left|\frac{x^{s}}{s}\right| d t & \ll \frac{\log \log T}{T} \int_{T / 2}^{T}\left|\zeta^{4}(1 / 2+i t)\right|\left|x^{s}\right| d t \\
& \ll(\log T)^{4}(\log \log T) x^{\sigma}
\end{aligned}
$$

Hence, we get

$$
\begin{aligned}
\int_{1 / 2}^{1} \int_{T / 2}^{T}\left|\frac{\zeta^{4}(\sigma+i t)}{\zeta(2 \sigma+2 i t)}\right|\left|\frac{x^{s}}{s}\right| d \sigma d t & \ll \int_{1 / 2}^{1} \int_{T / 2}^{T}\left|\zeta^{4}(\sigma+i t)\right|(\log \log T) x^{\sigma} \frac{d \sigma d t}{|t|} \\
& \ll(\log T)^{4}(\log \log T)\left(x-x^{1 / 2}\right)(\log x)^{-1}
\end{aligned}
$$

4. Proof of the Main Theorem. In Lemma 3.1, we take

and hence we obtain

$$
C=1+\frac{1}{\log x}, \quad f(s)=\frac{\zeta^{4}(s)}{\zeta(2 s)},
$$

$$
\begin{aligned}
& \frac{1}{T} \int_{0}^{T} \ldots \frac{1}{T} \int_{0}^{T}\left(\frac{1}{2 \pi i} \int_{1+1 / \log x-i T-i \phi}^{1+1 / \log x+i T+i \phi} \frac{\zeta^{4}(s)}{\zeta(2 s)} \frac{x^{s}}{s} d s\right) d \tau_{1} \ldots d \tau_{k} \\
= & \sum_{n \leq x} d^{2}(n)+\frac{\theta}{\pi} \sum_{n=1}^{\infty} d^{2}(n)\left(\frac{x}{n}\right)^{1+1 / \log x} \min \left(\pi+2+\frac{1+\log x}{T \log x}, M\right),
\end{aligned}
$$


where

$$
M=\min _{0 \leq m \in \mathbb{Z}^{+} \leq k}\left(\frac{2^{m+1}-1}{m+1}\right)|T \log (x / n)|^{-m-1},
$$

with $|\theta| \leq 1$. Now, we fix $m=0$ in (4.2) so that, from (4.1), we get

$$
\frac{1}{2 \pi i} \int_{1+1 / \log x-i T-i \phi}^{1+1 / \log x+i T+i \phi} \frac{\zeta^{4}(s)}{\zeta(2 s)} \frac{x^{s}}{s} d s=\sum_{n \leq x} d^{2}(n)+E+\theta_{1} x^{\varepsilon},
$$

where

$$
E=\frac{\theta}{\pi} \sum_{n=1}^{\infty} d^{2}(n)\left(\frac{x}{n}\right)^{1+1 / \log x} \min \left(\pi+2+\frac{1+\log x}{T \log x},\left|T \log \left(\frac{x}{n}\right)\right|^{-1}\right)
$$

and $\left|\theta_{1}\right| \leq 1$

Estimation of $E$. We choose $T=x^{1 / 2}$.

CAse (i). Suppose that $|x-n| \leq x^{\varepsilon}$. Then (since $\left.\pi+2+\frac{1+\log x}{T \log x} \leq 100\right)$, we obtain

$$
\left|E_{|x-n| \leq x^{\varepsilon}}\right| \leq \frac{100}{\pi} \sum_{|x-n| \leq x^{\varepsilon}} d^{2}(n) \ll x^{2 \varepsilon}
$$

CASE (ii). Suppose that $|x-n| \geq x / 2$. Therefore, we observe that

$$
\left|\log \left(\frac{x}{n}\right)\right|^{-1} \leq \frac{x}{|x-n|} \leq 10
$$

and hence

$$
\begin{aligned}
\left|E_{|x-n| \geq x / 2}\right| & \leq \frac{10}{T} \sum_{|x-n| \geq x / 2} d^{2}(n)\left(\frac{x}{n}\right)^{1+1 / \log x} \\
& \leq \frac{10}{T} \sum_{n=1}^{\infty} d^{2}(n)\left(\frac{x}{n}\right)^{1+1 / \log x} \ll \frac{x}{T}(\log x)^{4} .
\end{aligned}
$$

CAse (iii). Suppose that $x^{\varepsilon} \leq|x-n| \leq x / 2$. A result of Nair and Tenenbaum (see [11] and also [10]) states that

$$
\sum_{L \leq n \leq L+h} d^{2}(n) \ll h(\log L)^{3},
$$

for $h \geq L^{\varepsilon}$. We notice that

$$
\left(\frac{x}{n}\right)^{1+1 / \log x} \leq 4 \quad \text { for } n \in\left[x / 2, x-x^{\varepsilon}\right] \cup\left[x+x^{\varepsilon}, 3 x / 2\right] .
$$

Therefore, from (4.7), we have 


$$
\begin{aligned}
\left|E_{x^{\varepsilon} \leq|x-n| \leq x / 2}\right| & \leq \frac{100 x}{T} \sum_{x^{\varepsilon} \leq|x-n| \leq x / 2} d^{2}(n) \frac{1}{|x-n|} \\
& \leq \frac{100 x}{T} \sum_{U, U=2^{l} x^{\varepsilon}} \sum_{U \leq|x-n|<2 U} d^{2}(n) \frac{1}{U} \\
& \leq \frac{100 x}{T} \sum_{2 x \geq U \geq x^{\varepsilon}} \frac{1}{U} \sum_{n \in(x-2 U, x-U) \cup(x+U, x+2 U)} d^{2}(n) \\
& \ll \frac{x(\log x)^{4}}{T} .
\end{aligned}
$$

So, from (4.5), (4.6) and (4.8), we conclude that

$$
|E| \ll x^{\varepsilon}+\left(x(\log x)^{4}\right) / T
$$

for any small positive constant $\varepsilon$. Now, we choose a suitable horizontal line $t=t_{0} \in[T / 2, T]$ and we move the line of integration appearing in (4.3) to the line $\sigma=\sigma_{0}=1 / 2$ along $t=t_{0}$. We observe that the pole at $s=1$ (which is of order 4) contributes the main term and from Lemma 3.4, we observe that the horizontal portions contribute an error

$$
\ll \frac{x}{T}(\log T)^{4}(\log \log T)(\log x)^{-1} .
$$

Also, from Lemma 3.3, we observe that the vertical portion (with $s_{0}=$ $1 / 2+i t)$ in absolute value is

$$
\ll x^{1 / 2} \int_{|t| \leq T}\left|\frac{\zeta^{4}\left(s_{0}\right)}{\zeta\left(2 s_{0}\right)}\right|\left|\frac{d t}{s_{0}}\right| \ll x^{1 / 2}(\log T)^{5}(\log \log T) .
$$

Now, our choice $T=x^{1 / 2}$ proves the Main Theorem.

Acknowledgements. The authors are thankful to the referee for some useful comments on the earlier version of this paper. The authors are highly indebted to Professor K. Chandrasekharan for drawing their attention to the paper [5] and for his constant encouragement.

\section{References}

[1] R. Balasubramanian and K. Ramachandra, Some problems of analytic number theory, II, Studia Sci. Math. Hungar. 14 (1979), 193-202.

[2] - - - Some problems of analytic number theory, III, Hardy-Ramanujan J. 4 (1981), $13-40$.

[3] R. Balasubramanian, K. Ramachandra and M. V. Subbarao, On the error function in the asymptotic formula for the counting function of $k$-full numbers, Acta Arith. 50 (1988), 107-118.

[4] K. Chandrasekharan, Introduction to Analytic Number Theory, Grundlehren Math. Wiss. 148, Springer, New York, 1968. 
[5] K. Chandrasekharan and A. Good, On the number of integral ideals in Galois extensions, Monatsh. Math. 95 (1983), 99-109.

[6] A. Ivić, The Riemann Zeta-Function. The Theory of the Riemann Zeta-Function with Applications, Wiley, New York, 1985.

[7] M. Kühleitner, On a question of A. Schinzel concerning the sum $\sum_{n \leq x}(r(n))^{2}$, Grazer Math. Ber. 318 (1993), 63-67.

[8] M. Kühleitner and W. G. Nowak, An omega theorem for a class of arithmetic functions, Math. Nachr. 165 (1994), 79-98.

[9] H. L. Montgomery and R. C. Vaughan, The distribution of squarefree numbers, in: Recent Progress in Analytic Number Theory, 1 (Durham, 1979), Academic Press, London, 1981, 247-256.

[10] M. Nair, Multiplicative functions of polynomial values in short intervals, Acta Arith. 62 (1992), 257-269.

[11] M. Nair and G. Tenenbaum, Short sums of certain arithmetic functions, Acta Math. 180 (1998), 119-144.

[12] K. Ramachandra, On the Mean-Value and Omega-Theorems for the Riemann ZetaFunction, Lecture Notes Published by TIFR, Springer, Mumbai, 1995.

[13] -, A large value theorem for $\zeta(s)$, Hardy-Ramanujan J. 18 (1995), 1-9.

[14] - A remark on Perron's formula, J. Indian Math. Soc. 65 (1998), 145-151.

[15] - Notes on prime number theorem, I, in: Number Theory, R. P. Bambah, V. C. Dumir, R. J. Hans-Gill (eds.), Hindustan book Agency and Indian National Science Academy, 1999, 351-370.

[16] S. Ramanujan, Some formulae in the analytic theory of numbers, Messenger of Math. XLV (1916), 81-84.

[17] —, Ramanujan's Papers, B. J. Venkatachala et al. (eds.), Prism Books, 2000, 169-173.

[18] A. Sankaranarayanan and K. Srinivas, On a method of Balasubramanian and Ramachandra (on the abelian group problem), Rend. Sem. Mat. Univ. Padova 97 (1997), 135-161.

[19] A. Schinzel, On an analytic problem considered by Sierpiński and Ramanujan, in: New Trends in Probability and Statistics (Palanga, 1991), VSP, Utrecht, 1992, $165-171$.

[20] P. Shiu, A Brun-Titchmarsh theorem for multiplicative functions, J. Reine Angew. Math. 313 (1980), 161-170.

[21] E. C. Titchmarsh, The Theory of the Riemann Zeta-Function, 2nd ed. (edited by D. R. Heath-Brown), Clarendon Press, Oxford, 1986.

School of Mathematics

TIFR Centre

IISc Campus

Bangalore 560 012, India

E-mail: kram@math.tifrbng.res.in
School of Mathematics Tata Institute of Fundamental Research Homi Bhabha Road Mumbai 400 005, India E-mail: sank@math.tifr.res.in

Received on 26.4.2002

and in revised form on 17.10.2002 